\title{
Protocolo de estudo para avaliar programas de prevenção e controle de doenças cardiovasculares em uma empresa de petróleo
}

\author{
Study protocol to evaluate cardiovascular \\ disease prevention and control programs in an \\ oil company
}

\section{Maria Lúcia Ribeiro Rocha' ${ }^{1}$ Ana Marice Teixeira Ladeia ${ }^{2}$ (1)}

\begin{abstract}
${ }^{1}$ Autora para correspondência. Empresa Brasileira de Serviços Hospitalares/EBSERH (Salvador), Escola Bahiana de Medicina e Saúde Pública (Salvador). Bahia, Brasil. mluciarocha@bahiana.edu.br ${ }^{2}$ Escola Bahiana de Medicina e Saúde Pública (Salvador). Bahia, Brasil. anamarice@bahiana.edu.br
\end{abstract}

RESUMO I INTRODUÇÃO: Profissionais de saúde em empresas têm implementado programas de prevenção e controle de doenças crônicas não transmissíveis (DCNT) no intuito de reduzir adoecimento e mortes de empregados. Estes programas diferem na metodologia adotada, não havendo na literatura dados sobre a estratégia que oferece o melhor custo benefício. OBJETIVOS: Avaliar se o modelo interdisciplinar de um programa de prevenção e controle de DCNT é superior ao modelo multidisciplinar na redução de risco cardiovascular (RCV) em empregados de uma empresa de petróleo e descrever o perfil de saúde de empregados desta empresa. MÉTODOS: Estudo analisará uma coorte retrospectiva de empregados de uma empresa de petróleo da Bahia, com dados do período de 01.01.2016 a 31.12.2018. A amostra disponível foi dividida em dois grupos, de acordo com o programa ao qual o empregado está vinculado. Foram coletados dados de saúde em prontuário eletrônico, os quais serão analisados utilizando o pacote estatístico SPSS versão 12.0. O impacto dos programas será verificado comparando a frequência da variável RCV entre os anos 2016 e 2018, por meio dos testes McNemar e Qui-quadrado, com P $<5 \%$. Estudo aprovado através de CAAE 14107619.3.0000.5544. RESULTADOS ESTIMADOS: Resultados deste estudo possibilitarão o redirecionamento de ações por parte desta empresa, considerando questões técnicas, científicas e econômicas envolvidas, no intuito de atingir de forma efetiva um maior número de pessoas. Outras empresas poderão vir a adotar o modelo mais adequado para reduzir o RCV entre seus empregados, colaborando com a redução de mortes por este grupo de doenças no Brasil.

PALAVRAS-CHAVE: Risco cardiovascular. Programas de prevenção. Doenças cardiovasculares.
ABSTRACT | INTRODUCTION: Health professionals in companies have implemented programs for the prevention and control of chronic noncommunicable diseases (CNCDs) in order to reduce illness and deaths of employees. These programs differ in the methodology adopted, with no data in the literature on the strategy that offers the best cost benefit. OBJECTIVES: To assess whether the interdisciplinary model of a CNCDs prevention and control program is superior to the multidisciplinary model in cardiovascular risk reduction (CVR) in workers of an oil company and to describe the health profile of workers of the company. METHODS: The study will analyze a retrospective cohort of employees of an oil company in Bahia, with data from the period from 01.01.2016 to 12.31.2018. The available sample was divided into two groups, according to the program to which the employee was allocated. Health data were collected from electronic medical records, which will be analyzed using the SPSS version 12.0 statistical package. The impact of the programs will be verified by comparing the frequency of the RCV variable between the years 2016 and 2018, using the McNemar and Chi-square tests, with $\mathrm{P}<5 \%$. This study was approved under protocol CAAE 14107619.3.0000.5544. PREDICTED RESULTS: Results of this study should allow the redirection of actions by this company, considering technical, scientific and economic issues involved, in order to effectively reach a larger number of people. Other companies may adopt the most appropriate model to reduce CVR among their employees, helping to reduce deaths from this group of diseases in Brazil.

KEYWORDS: Cardiovascular risk. Prevention programs. Cardiovascular diseases. 


\section{Introdução}

O perfil de saúde da população tem se modificado ao longo dos tempos, associado a fatores como avanços tecnológicos, desenvolvimento econômico e educacional, crescimento demográfico, impondo redirecionamento das políticas sociais e das estratégias de promoção, prevenção e controle de doenças ${ }^{1-4}$.

Segundo a Organização Pan Americana da Saúde (OPAS) em 2016 as doenças crônicas não transmissíveis (DCNT) foram responsáveis por $71 \%$ de todas as mortes no mundo, sendo que mais de $85 \%$ de mortes prematuras ocorreram em países de baixa e média renda, associadas às desigualdades socioeconômicas existentes ${ }^{5-7}$. No Brasil, em 2018, 54\% do total de óbitos foi por DCNT, segundo dados do Ministério da Saúde ${ }^{8}$.

Diante desta realidade, as DCNT tornaram-se grande problema de saúde pública, determinando a necessidade de elaboração de estratégias de enfrentamento por parte de órgãos e instituições que atuam na área de saúde em todo o mundoq-15.

As empresas, por manterem como empregados indivíduos na fase produtiva, mais sujeitos ao adoecimento por DCNT, possuem importante papel no controle dos fatores de risco modificáveis, já que por determinação legal acompanham a saúde destas pessoas anualmente ${ }^{16}$.

Dados da literatura apontam que empresas tem adotado ações de redução do risco de DCNT, entretanto informações relacionadas à comparação da efetividade obtida por diferentes estratégias são escassos ${ }^{17-20}$.
Este trabalho oferecerá os resultados obtidos com a implementação de dois programas de intervenção em uma empresa de petróleo, que apresentam como objetivo a redução do risco cardiovascular (RCV) entre empregados acompanhados por profissionais da área de saúde ocupacional, utilizando recursos e estratégias diferentes. Como objetivo principal será testada a hipótese de que o modelo interdisciplinar de um programa de prevenção e controle de DCNT apresenta resultado superior ao modelo multidisciplinar na redução do risco cardiovascular em empregados de uma empresa de petróleo, além de descrever o perfil de saúde destes empregados para o período de 2016 a 2018.

Com estas informações será possível redirecionar ações, considerando aspectos técnicos, científicos e econômicos envolvidos, no intuito de atingir de forma efetiva um maior número de pessoas, contribuindo com a redução do absenteísmo médico, o que pode vir a repercutir positivamente nos resultados do negócio. Outras empresas poderão vir a adotar o modelo mais adequado para reduzir o RCV entre seus empregados, colaborando com a redução de mortes por este grupo de doenças no Brasil.

\section{Métodos}

\section{Desenho do Estudo}

Estudo de coorte retrospectiva de população restrita e fixa, cujas informações foram incluídas no prontuário eletrônico no período de 01 de janeiro de 2016 a 31 de dezembro de 2018. 


\section{Local}

Empresa de petróleo na Bahia, com Serviços de Saúde Ocupacional em Salvador e na base operacional de São Sebastião do Passé.

\section{População Alvo}

Empregados ativos no período de 2016 a 2018.

\section{Seleção da Amostra}

Amostra disponível, composta por empregados que preencherem os critérios de inclusão, os quais foram divididos em dois grupos, de acordo com o tipo de programa ao qual estava vinculado. Empregados acompanhados por equipe de Salvador farão parte do grupo A (modelo multidisciplinar/controle) e os que participaram do programa na base operacional comporão o grupo B (modelo interdisciplinar/caso).

\section{Critérios de Inclusão}

a) Estar vinculado à empresa no período de 1.01.2016 a 31.12.2018;

b) Ter realizado avaliação médica para cada ano avaliado;

c) Ter permanecido no mesmo cargo/função, no mesmo local de trabalho e no mesmo regime durante o período estudado;

d) Possuir registro em prontuário da pressão arterial, peso e altura para cada ano estudado, assim como resultado do colesterol total, colesterol $\mathrm{HDL}$, colesterol LDL, triglicérides e a glicemia em jejum.

\section{Critérios de exclusão}

a) Indivíduo afastado da empresa por mais de quatro meses, em um ano, por motivo de doença, exceto DCV; b) Indivíduo com diagnóstico de patologia que possa distorcer o cálculo do risco cardiovascular, como neoplasias, transtorno psiquiátrico, doenças da tireoide, uso prolongado de corticosteroides, etc.

\section{Protocolo do Estudo}

Serão analisados dados já coletados de empregados que estavam ativos no período de 01.01.2016 a 31.12.2018, obtidos do prontuário médico eletrônico. A renda foi obtida da tabela do Plano de Cargos e Salários da empresa, considerando a faixa atribuída ao cargo, sem considerar adicionais por tempo de serviço ou remunerações por função gratificada.

Dados do prontuário resultam de avaliações médicas realizadas anualmente pelos empregados e contemplam informações clínicas, ocupacionais e exames complementares.

A pressão arterial foi obtida por aparelhos de esfignomanômetro manual ou digital, calibrados pelo INMETRO, com o indivíduo sentado em cadeira com dorso, pés apoiados no chão, membro superior esquerdo sobre a mesa.

O peso foi verificado em balanças digitais ou analógicas, calibradas, com indivíduo utilizando a roupa de trabalho, sem sapatos, registrado em quilo, com duas casas decimais. A altura foi referida pelo indivíduo ou obtida com o emprego do estadiômetro, em centímetros, com o indivíduo de pé e descalço. O IMC foi obtido a partir da fórmula peso/altura².

Os exames laboratoriais foram realizados com o indivíduo em jejum, em clínicas conveniadas.

Critérios adotados para o diagnóstico de obesidade, dislipidemias, hipertensão arterial e diabetes foram aqueles estabelecidos em diretrizes das respectivas sociedades científicas ${ }^{21-24}$ descritos no quadro 1. 
Quadro 1. Critérios para diagnóstico de doenças metabólicas

\begin{tabular}{|c|c|}
\hline INDICADOR & CRITÉRIO \\
\hline $\mathrm{IMC}^{21}$ & \\
\hline Normal & $18,5 \mathrm{Kg} / \mathrm{m}^{2}$ e $24,9 \mathrm{Kg} / \mathrm{m}^{2}$ \\
\hline Sobrepeso & $25 \mathrm{Kg} / \mathrm{m}^{2}$ a $29,9 \mathrm{Kg} / \mathrm{m}^{2}$ \\
\hline Obesidade Grau I & $30,0 \mathrm{Kg} / \mathrm{m}^{2}$ a $34,9 \mathrm{Kg} / \mathrm{m}^{2}$ \\
\hline Obesidade Grau II & $35,0 \mathrm{Kg} / \mathrm{m}^{2}$ a $39,9 \mathrm{Kg} / \mathrm{m}^{2}$ \\
\hline Obesidade Grau III & $40,0 \mathrm{Kg} / \mathrm{m}^{2}$ ou mais \\
\hline Circunferência Abdominal & $94 \mathrm{~cm}$ em homens; $80 \mathrm{~cm}$ em mulheres \\
\hline Hipercolesterolemia isolada ${ }^{22}$ & $\mathrm{LDL}-\mathrm{c}=160 \mathrm{mg} / \mathrm{dL}$ \\
\hline Hipertrigliceridemia isolada & $\mathrm{TG}=150 \mathrm{mg} / \mathrm{dL}$ \\
\hline Hiperlipidemia mista & $\mathrm{LDL}-\mathrm{c}=160 \mathrm{mg} / \mathrm{dL}$ e dos $\mathrm{TG}=150 \mathrm{mg} / \mathrm{dL}$ \\
\hline Colesterol total & $\mathrm{CT}>190 \mathrm{mg} / \mathrm{dl}$ \\
\hline HDL-c baixo & $\mathrm{HDL}-\mathrm{c}<40 \mathrm{mg} / \mathrm{dL}$ (homens) $\mathrm{HDL}-\mathrm{c}<50 \mathrm{mg} / \mathrm{dL}$ (mulheres) \\
\hline $\begin{array}{l}\text { Hipertensão Arterial }^{23} \\
\text { Pré-hipertenso }{ }^{23}\end{array}$ & $\begin{array}{l}\text { PA =140 x } 90 \mathrm{mmHg} \text { no consultório, ou diagnóstico estabelecido } \\
\text { PA sistólica (PAS) entre } 121 \text { e } 139 \quad \mathrm{mmHg} \text { e/ou PA diastólica } \\
\text { (PAD) entre } 81 \text { e } 89 \mathrm{mmHg} \text {, no consultório }\end{array}$ \\
\hline Pré-diabetes & $\begin{array}{l}\text { Hemoglobina glicada (HbA 1c) }=6,5 \% \text { ou Glicemia de jejum }=126 \\
\mathrm{mg} / \mathrm{dl}(7,0 \mathrm{mmol}) \\
\text { Hemoglobina glicada (HbA } 1 \mathrm{c}) \text { entre } 5,7 \text { e } 6,4 \% \text { ou Glicemia em } \\
\text { jejum entre } 100 \mathrm{mg} / \mathrm{dl} \mathrm{e} 125 \mathrm{mg} / \mathrm{dl}\end{array}$ \\
\hline
\end{tabular}

O risco cardiovascular foi calculado segundo o escore de Framingham 25,26 , que indica a probabilidade de ocorrência de eventos cardiovasculares em 10 anos, utilizando como variáveis idade, sexo, tabagismo, glicemia, pressão arterial sistólica, colesterol total e o HDL-C. Os indivíduos serão distribuídos de acordo com a classificação de risco em baixo $(<10 \%)$, moderado $(>10 \%$ e $<20 \%)$ e alto $(>20 \%)$.

As variáveis comportamentais serão categorizadas de acordo com os critérios estabelecidos no quadro 2. 
Quadro 2. Categorização de variáveis comportamentais

\begin{tabular}{|c|c|c|}
\hline VARIÁVEL & AVALIAÇÄO & CLASSIFICAÇÄO \\
\hline Uso de Tabaco & $\begin{array}{l}\text { Ex fumante } \\
\text { Fumante } \\
\text { Não fum ante } \\
\text { Fum ante passivo } \\
\text { Experimentador }\end{array}$ & $\begin{array}{l}\text { Deixou de fumar e não retorno } \\
\text { Fumou }>100 \text { cigarros } N i d a \\
\text { fuma; } \\
\text { Nunca fumou; } \\
\text { Não fuma e convive com fuman } \\
\text { Fumou }<100 \text { cigarros } / \text { ida e fur }\end{array}$ \\
\hline Uso de álcool & $\begin{array}{l}\text { Todos os dias; } \\
1 \text { a } 4 \text { vezes na semana; } \\
01 \text { a } 03 \text { vezes no mês; } \\
\text { Menos de } 01 \text { vez/mês; } \\
\text { Menos de } 01 \text { vez/ano ou nun } \\
\text { bebeu. }\end{array}$ & $\begin{array}{l}\text { Frequente pesado; } \\
\text { Frquente; } \\
\text { Menos frequente; } \\
\text { Não frequente; } \\
\text { Abstêmio. }\end{array}$ \\
\hline $\begin{array}{l}\text { Dieta } \\
\text { (consumo de } \\
\text { FLV) }\end{array}$ & $\begin{array}{l}\text { Número de refeições dia com } \\
\text { consumo de frutas, legumes } \\
\text { e verduras }\end{array}$ & $\begin{array}{l}\text { 1- Não consome } \\
\text { 2- Muito baixo - } 1 \\
\text { 3- Baixo - } 2 \text { a } 3 \\
\text { 4- Regular - } 4 \\
\text { 5- Bom - } 5\end{array}$ \\
\hline $\begin{array}{l}\text { Nivel de } \\
\text { atividade } \\
\text { física }\end{array}$ & $\begin{array}{l}\text { Tipo de atividade (leve, } \\
\text { moderada ou vigorosa), dias } \\
\text { na semana; tempo de } \\
\text { prática/dia }\end{array}$ & $\begin{array}{l}\text { Inativo; } \\
\text { Irregularmente ativo; } \\
\text { Regularmente ativo; } \\
\text { Muito ativo }\end{array}$ \\
\hline
\end{tabular}

Fonte: Sistema informatizado corporativo de saúde

\section{Modelos de Programas de Intervenção em Saúde}

Dois programas de prevenção e controle de DCNT foram implementados na empresa, os quais diferem em metodologia. O modelo multidisciplinar (controle) foi desenvolvido pela equipe de saúde de Salvador, enquanto que o interdisciplinar (caso) foi implementado na base operacional de São Sebastião do Passé.

\section{A. Modelo Multidisciplinar (Controle)}

Empregados após realizarem exames complementares em serviços credenciados agendavam a avaliação periódica, a qual iniciava com o técnico de enfermagem verificando dados antropométricos, pressão arterial e o estado de imunização. Posteriormente o empregado era avaliado por médico, odontologista e nutricionista. Cada profissional, independentemente, orientava o empregado de acordo com problemas identificados e estimulavam a adoção de hábitos saudáveis, como a prática de atividade física, alimentação saudável, redução do uso de tabaco, álcool, ou outras drogas (Figura 1). Não havia discussão de casos entre os profissionais que compõe a equipe de saúde. 


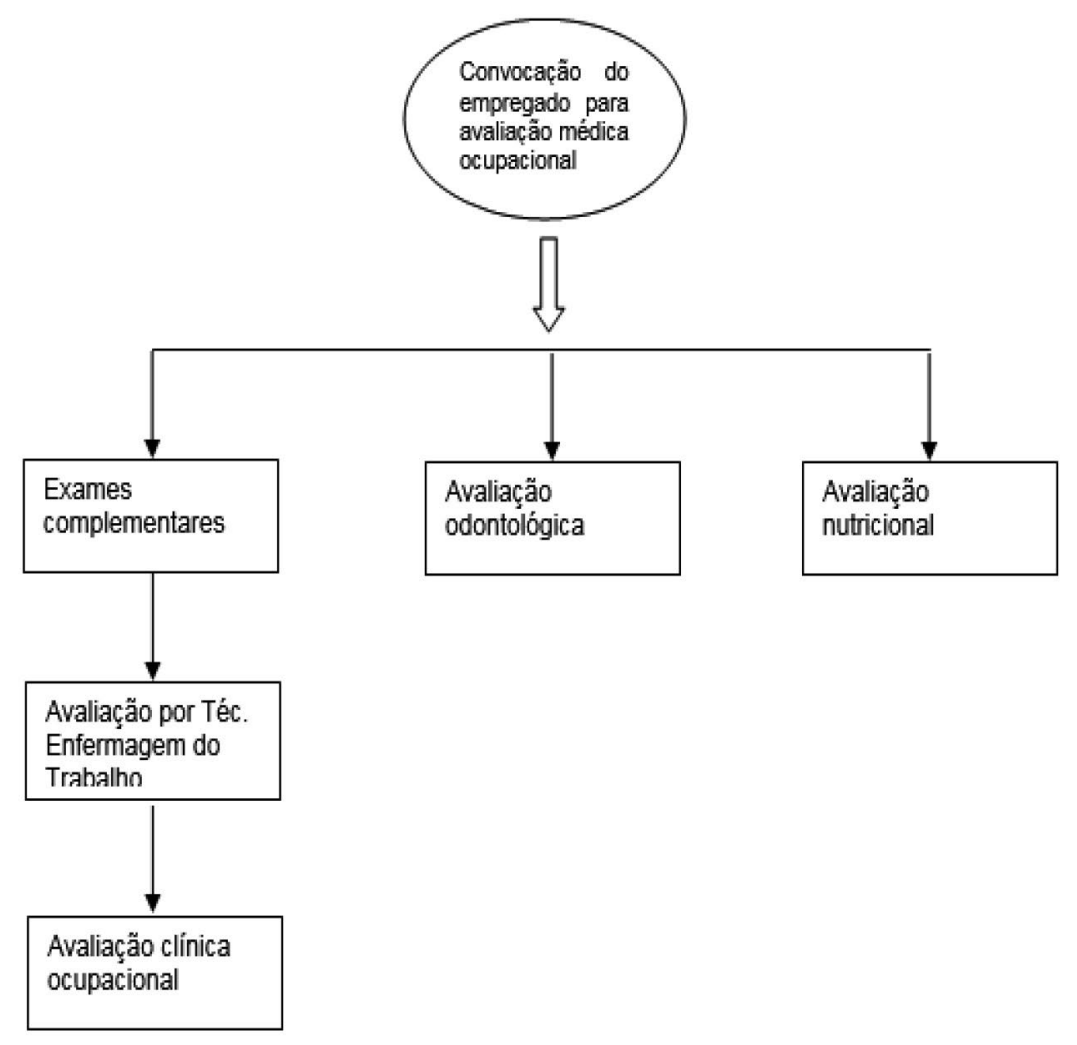

As refeições destes empregados eram realizadas fora do local de trabalho e a empresa patrocinava a realização de atividade física em academias externas para os interessados, desde que se submetessem às avaliações semestrais com o educador físico.

\section{B. Modelo Interdisciplinar (Caso)}

$\mathrm{Na}$ área operacional o programa teve como proposta a interdisciplinaridade de ações realizadas por profissionais de saúde. Após a conclusão de exames complementares em serviços credenciados o empregado comparecia ao Serviço de Saúde Ocupacional para a avaliação periódica previamente agendada. Esta iniciava com o educador físico, prosseguia com o médico, nutricionista, enfermeiro, odontólogo e técnico de higiene bucal, os quais forneciam recomendações de acordo com problemas identificados, com foco no autocuidado. Ao final do processo um profissional de saúde, enfermeiro ou psicólogo, discutia todos os problemas identificados por cada profissional que havia realizado o atendimento e reforçava a necessidade da adoção de hábitos de vida saudáveis e da submissão a tratamentos, quando necessários.
Após os atendimentos cada caso era discutido pela equipe e um plano de acompanhamento ao empregado era estabelecido de acordo com o grau de risco para DCV, segundo o Escore de Framingham ${ }^{25,26}$, que incluía agendamento do retorno e encaminhamento a especialistas, se necessário. A partir desta fase ficava definido se o empregado seria atendido, também, por fisioterapeuta, assistente social e psicóloga, a depender da necessidade de saúde que apresentasse. As ações instituídas estavam voltadas para o controle de patologias crônicas e redução de fatores de risco para DCNT, além do acompanhamento de pessoas com transtorno de ansiedade, depressão ou dor crônica. Como estratégia havia o estímulo ao consumo de frutas, legumes e verduras e à adoção da prática de atividade física (Figura 2).

Este programa oferecia ao empregado espaço interno para a prática de exercícios físicos por trinta minutos da sua jornada de trabalho, ou subsidiava a frequência em espaços externos à unidade. Os participantes eram avaliados por educador físico antes de iniciar o programa e semestralmente, independentemente da classificação de risco para DCV que apresentasse. 
Os empregados acompanhados no programa eram discutidos em reuniões interdisciplinares mensais, quando decisões relacionadas às necessidades de intervenção individual eram estabelecidas. A equipe tinha como meta reduzir o risco de saúde de cada participante, sendo os indicadores do programa apresentados e discutidos em reuniões de análise crítica gerencial.

Figura 2. Fluxograma do atendimento por equipe interdisciplinar

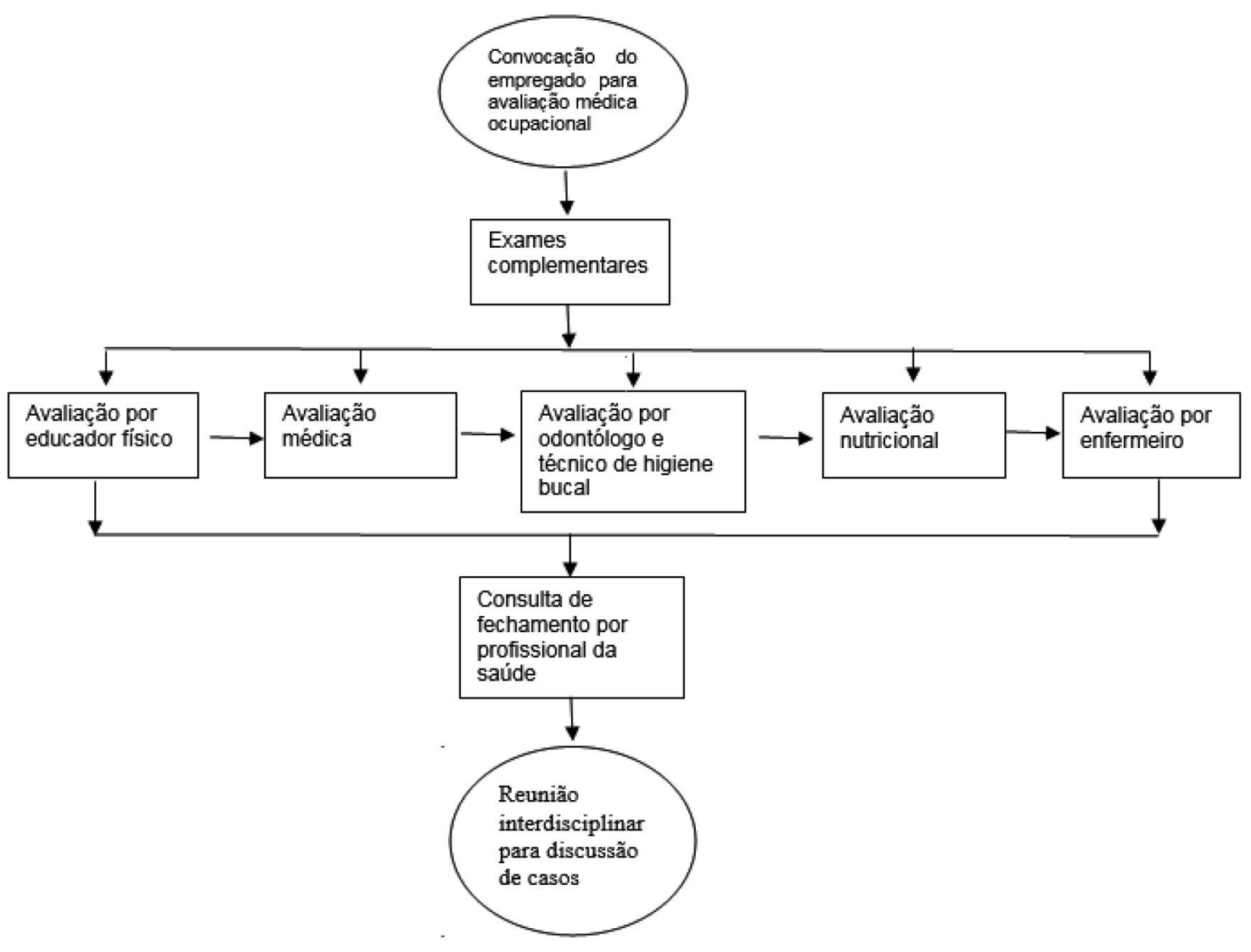

Empregados de áreas operacionais recebiam alimentação da empresa, preparada de acordo com cardápio supervisionado por nutricionista da equipe de saúde, o qual previa a redução da oferta de gorduras e carboidratos simples, assim como o aumento da oferta de fibras.

Vale ressaltar que o empregado não estava obrigado a aderir ao programa e que não havia prejuízo administrativo previsto para casos em que o plano de acompanhamento proposto não era cumprido. Entretanto, quando o estado de saúde era incompatível com as exigências das tarefas ocorria afastamento temporário ou permanente das atividades.

\section{Hipóteses Estatísticas}

Hipótese Nula (HO): Os programas multidisciplinar e interdisciplinar não apresentam diferenças significativas de resultados na redução do risco cardiovascular.

Hipótese Alternativa (HA): O programa interdisciplinar apresenta resultado superior na redução do risco cardiovascular ao obtido pelo modelo multidisciplinar. 


\section{Variáveis}

Empregados serão analisados de acordo com idade, sexo, escolaridade, renda, local de trabalho, tempo de empresa, regime de trabalho, tipo de atividade desenvolvida (operacional, administrativa, gerencial), peso, altura, índice de massa corporal (IMC), circunferência abdominal, pressão arterial, glicemia de jejum, colesterol total, HDL, LDL, risco cardiovascular, nível de atividade física, consumo de frutas, legumes e verduras.

\section{Plano de Análise Estatística}

A análise de dados será realizada utilizando o pacote estatístico SPSS (Statistical Package for the Social Sciences) versão 12.0, no intuito de testar a hipótese de que o modelo interdisciplinar (caso) do programa de prevenção e controle de DCNT obteve resultado superior na redução do risco cardiovascular ao do modelo multidisciplinar (controle), desenvolvidos em uma empresa de petróleo.

O cálculo do tamanho da amostra obteve um $\mathrm{N}$ de 118 indivíduos para cada grupo, com base na regra de proporções, que considerou que após a intervenção o grupo interdisciplinar passou a ter $20 \%$ de indivíduos com risco cardiovascular alto, enquanto o grupo multidisciplinar teria 40\%, para demonstrar a diferença entre os grupos, com valor de a de 0,05 e $\beta$ de $80 \%$. Contudo, pretende-se que a amostra seja composta pela totalidade de empregados da empresa, desde que preencham os critérios de inclusão. Em 31 de dezembro de 2018 este grupo era composto por 880 pessoas, sendo 646 em áreas operacionais e 234 em Salvador.

A diferença de impacto entre os dois programas será verificada comparando a frequência do risco cardiovascular alto, segundo escore de Framingham $\underline{25} \underline{26}$, entre os anos de 2016 e 2018, apresentada pelos empregados dos grupos A (multidisciplinar/ controle) e B (interdisciplinar/caso), por meio do teste do Quiquadrado (entre grupos, variáveis categóricas).

O impacto de cada programa será verificado comparando a frequência do risco cardiovascular, segundo escore de Framingham 25,26 , apresentada pelos empregados de cada grupo, nos anos de 2016 e 2018, por meio do teste de McNemar (intra-grupo, pareado), para observar se houve redução no percentual de indivíduos classificados como risco alto.
Os grupos serão comparados para os anos de $2016 \mathrm{e}$ 2018, intra e entre grupos, para as variáveis relacionadas à saúde e comportamentais por meio do teste de McNemar (intra-grupo, pareado, variáveis categóricas) e do Qui-quadrado (entre grupos, variáveis categóricas), teste $T$ de Student para comparar médias (variáveis numéricas).

O perfil de saúde dos empregados será descrito para o período de 2016 a 2018, segundo as variáveis demográficas, comportamentais, ocupacionais e de saúde, com utilização de média e desvio padrão (dp) para variáveis numéricas e frequência para variáveis categóricas, para verificar a tendência no período estudado.

Será verificado se existe diferença na distribuição dos fatores de risco e do risco cardiovascular alto segundo escore de Framingham 25,26 por faixa etária, sexo, cargo, áreas de trabalho, regime de trabalho, prática de atividade física e alimentação. Análise de regressão logística será realizada para verificar efeito de confusão destas variáveis, considerando como desfecho o risco cardiovascular alto.

As análises estatísticas serão realizadas assumindose o nível de significância de $5 \%(p<0,05)$ para todos os testes utilizados.

\section{Exequibilidade}

O estudo utilizará dados de prontuário eletrônico, aos quais uma das pesquisadoras (MLRR) tem acesso por ser médica da empresa, com anuência da Médica Coordenadora do Programa de Controle Médico de Saúde Ocupacional (PCMSO) e da responsável legal da empresa.

A empresa apoia o projeto e terá benefícios relacionados à verificação da efetividade de ações desenvolvidas na prevenção e controle de fatores de risco de DCV, podendo adequar o planejamento do programa para otimizar recursos e melhorar resultados alcançados.

\section{Riscos}

Esta pesquisa será realizada a partir de dados secundários, o que resulta em risco mínimo aos participantes, segundo critérios da Resolução CNS 466/2012. 
Não haverá divulgação da identidade dos participantes, sendo o nome substituído por registro numérico, impossibilitando a associação de dados a pessoas.

Os dados serão tratados por médicos, os quais estão submetidos ao código de ética da categoria profissional, tendo o compromisso de salvaguardar a privacidade dos indivíduos.

\section{Benefícios}

Resultados deste estudo serão publicados em forma de artigo, contribuindo com a produção de conhecimentos na área de saúde pública, mais especificamente na área de saúde ocupacional, possibilitando que ações de prevenção e controle de fatores de risco para DCV possam ser revistas e ou adotadas por empresas de vários segmentos, beneficiando um grande número de trabalhadores. Com isso espera-se que ocorra redução do número de pessoas que adoecem ou morrem por esta causa.

Na empresa avaliada, o estudo será utilizado para definir diretrizes únicas para a área de saúde, ampliando o cuidado com empregados e otimizando recursos humanos e financeiros.

\section{Aspectos Éticos}

Estudo será realizado a partir de dados secundários, sem que haja necessidade de intervenções adicionais às já realizadas para avaliar a capacidade laboral dos empregados, conforme exigências legais.

Empregados que se encontram na empresa serão contatados e solicitados a preencher o Termo de Consentimento Livre e Esclarecido (TCLE).

Dados serão tratados por médicos, pesquisadores vinculadosaesteprojetocom CAAE 14107619.3.0000.5544, os quais respeitarão a privacidade dos sujeitos sob investigação, cuidando para que não ocorra associação de informações a indivíduos.

O acesso ao prontuário teve a anuência da Médica Coordenadora do PCMSO da empresa.

\section{Resultados Esperados}

A análise dos dados resultantes do monitoramento da saúde de empregados acompanhados em programas de prevenção permite avaliar a efetividades das ações desenvolvidas, identificando pontos de meIhoria, os quais serão considerados no planejamento para períodos subsequentes. Esta é uma prática recomendada em saúde pública, muito embora não seja frequente a comparação de resultados obtidos por diferentes metodologias, notadamente no campo da saúde ocupacional.

São escassas as publicações que avaliam diferentes modelos de intervenção, sobretudo com população fixa. Viterbo 27 publicou estudo comparando resultados de um programa de prevenção para DCNT interdisciplinar, com dados de empregados de diversas áreas de uma companhia de petróleo, sem acompanhamento sistemático pela área de saúde, sendo as coortes dinâmicas.

Este estudo proporcionará novos conhecimentos sobre o impacto de programas de saúde, já que apontará resultados da comparação de dois programas de prevenção desenvolvidos numa mesma empresa, com uma população fixa para o período estudado. Este cuidado reduzirá vieses relacionados às saídas e entradas na coorte, preenchendo lacunas epidemiológicas existentes.

Profissionais da área de saúde ocupacional terão como comparar as metodologias que têm adotado, com as propostas discutidas neste estudo, descobrindo novas formas de abordagem e de intervenção em saúde, com foco na redução de fatores de risco para DCV.

Este estudo não tem como proposta a avaliação do custo benefício dos programas, porém os dados obtidos poderão ser utilizados no desenvolvimento de futuras intervenções, no intuito de identificar qual das estratégias oferecem resultados que justificam os investimentos realizados em saúde. 


\section{Contribuições das autoras}

Rocha MLR elaborou o protocolo. Ladeia AMT realizou a revisão crítica e aprovou o texto final.

\section{Conflitos de interesses}

Não há conflitos de interesses relacionados ao financiamento do projeto ou ao tipo de resultado esperado. A pesquisadora, apesar de pertencer à empresa estudada, não fez parte das equipes relacionadas aos programas avaliados. A empresa não influenciou o desenho do estudo e nem influenciará na análise de dados. Nenhum outro conflito financeiro, legal ou político envolvendo terceiros (governo, empresas e fundações privadas, etc.) foi declarado para nenhum aspecto do trabalho submetido (incluindo, mas não se limitando a subvenções e financiamentos, participação em conselho consultivo, desenho de estudo, preparação de manuscrito, análise estatística, etc.).

\section{Referências}

1. Brasil. Ministério da Saúde. Secretaria de Vigilância em Saúde. Departamento de Análise de Situação de Saúde. Plano de Ações Estratégicas para o Enfrentamento das Doenças Crônicas Não Transmissíveis (DCNT) no Brasil 2011-2022. Brasília: Ministério da Saúde; 2011.

2. Instituto Brasileiro de Geografia e Estatística. Projeções e estimativas da população do Brasil e das Unidades da Federação [Internet]. [acesso em 2019 fev. 03].Disponível em: https://www. ibge.gov.br/apps/populacao/projecao/

3. Mathers CD, Loncar D. Projections of Global Mortality and Burden of Disease from 2002 to 2030. PLoS Med. 2006;3(11):e442. doi: $10.1371 /$ journal.pmed.0030442

4. Araújo JD. Polarização epidemiológica no Brasil. Epidemiol Serv Saúde. 2012;21(4):533-538. doi: 10.5123/S167949742012000400002

5. Organização Mundial de Saúde. Décimo terceiro programa geral de trabalho 2019-2023 [Internet]. [acesso em 2019 fev. 2]. Disponível em: http://apps.who.int/gb/ebwha/pdf_files/WHA71/ A71_4-en.pdf?ua=1

6. Achutti A. Prevenção de doenças cardiovasculares e promoção da saúde. Ciênc Saúde Coletiva. 2012;17(1):18-20. doi: 10.1590/ S1413-81232012000100003

7. Ishitani LH, Franco GC, Perpétuo IHO, França E. Desigualdade social e mortalidade precoce por doenças cardiovasculares no Brasil. Rev Saúde Pública. 2006;40(4):684-91. doi: 10.1590/5003489102006000500019
8. Brasil. Ministério da Saúde. DATASUS [Internet]. [acesso em 2019 fev. 2]. Disponível em: http://tabnet.datasus.gov.br/cgi/ tabcgi.exe?sim/cnv/obt10uf.def

9. Malta DC, Morais Neto OL, Silva Junior JB. Apresentação do plano de ações estratégicas para o enfrentamento das doenças crônicas não transmissíveis no Brasil, 2011 a 2022. Epidemiol Serv Saúde. 2011;20(4):425-438. doi: 10.5123/S167949742011000400002

10. Ribeiro AG, Cotta RMM, Ribeiro SMR. A promoção da saúde e a prevenção integrada de fatores de risco para doenças cardiovasculares. Ciênc. Saúde Coletiva. 2012;17(1):7-17. doi: 10.1590/S1413-81232012000100002

11. Brasil. Ministério da Saúde. Agência Nacional de Saúde Suplementar. Manual técnico para promoção da saúde e prevenção de riscos e doenças na saúde suplementar [Internet]. 4. ed. rev. e atual. Rio de Janeiro: Agência Nacional de Saúde Suplementar; 2011. Disponível em: http://www.ans.gov.br/ images/stories/Materiais_para_pesquisa/Materiais_por_assunto/ manual_promoprev_web.pdf.

12. Brasil. Ministério da Saúde. Secretaria de Atenção à Saúde. Departamento de Atenção Básica. Estratégias para o cuidado da pessoa com doença crônica: hipertensão arterial sistêmica [Internet]. Brasília: Ministério da Saúde; 2013. Disponível em http://bvsms.saude.gov.br/bvs/publicacoes/estrategias_cuidado_ pessoa_doenca_cronica.pdf

13. Malta DC, Silva Júnior JB. O Plano de Ações Estratégicas para o Enfrentamento das Doenças Crônicas Não Transmissíveis no Brasil e a definição das metas globais para o enfrentamento dessas doenças até 2025: uma revisão. Epidemiol Serv Saúde. 2013;22(1):151-164. doi: 10.5123/S1679-49742013000100016

14. Murray CJL, Lopez AD. Alternative projections of mortality and disability by cause 1990-2020: Global Burden of Disease Study. The Lancet. 1997;349(9064). doi: 10.1016/S0140-6736(96)07492-2

15. Brasil. Ministério da Saúde. Secretaria de Vigilância em Saúde. Departamento de Vigilância de Doenças e Agravos não Transmissíveis e Promoção da Saúde. Vigitel Brasil 2017: vigilância de fatores de risco e proteção para doenças crônicas por inquérito telefônico: estimativas sobre frequência e distribuição sociodemográfica de fatores de risco e proteção para doenças crônicas nas capitais dos 26 estados brasileiros e no Distrito Federal em 2017 [Internet]. Brasília: Ministério da Saúde; 2018. Disponível em: https://bvsms.saude.gov.br/bvs/publicacoes/ vigitel_brasil_2017_vigilancia_fatores_riscos.pdf

16. Brasil. Ministério da Economia. Secretaria do Trabalho. Escola Nacional de Inspeção do Trabalho. Segurança e Saúde no Trabalho. Normatização - Normas Regulamentadoras. Norma Regulamentadora número 7 - Programa de Controle Médico em Saúde Ocupacional (PCMSO) [Internet]. Disponível em: https://enit.trabalho.gov.br/portal/index.php/segurancae-saude-no-trabalho/sst-menu/sst-normatizacao/sst-nrportugues?view=default 
17. Matos MFD, Silva NAS, Pimenta AJM, Cunha AJLA. Prevalência dos Fatores de Risco para Doença Cardiovascular em Funcionários do Centro de Pesquisas da Petrobras. Arq Bras Cardiol. 2004;82(1):1-4. doi: 10.1590/S0066-782X2004000100001

18. Matos MFD, Fiszman R. Estratégias de prevenção para doenças cardiovasculares e promoção de saúde. Rev SOCERJ. 2003;16(2):133-140.

19. Ciorlia LAS, Godoy MF. Fatores de Risco Cardiovascular e Mortalidade. Seguimento em Longo Prazo (até 20 anos) em Programa Preventivo Realizado pela Medicina Ocupacional. Arq Bras Cardiol. 2005;85(1):20-25. doi: 10.1590/S0066782X2005001400005

20. Rocha RS, Conti RAS. Risco cardiovascular: abordagem dentro da empresa. Rev Bras Med Trab. 2005;3(1):10-21.

21. Associação Brasileira para o Estudo da Obesidade e da Síndrome Metabólica. Diretrizes brasileiras de obesidade [Internet]. 4.ed. São Paulo, SP: ABESO. Disponível em: https:// abeso.org.br/wp-content/uploads/2019/12/Diretrizes-DownloadDiretrizes-Brasileiras-de-Obesidade-2016.pdf

22. Sociedade Brasileira de Cardiologia. Atualização da Diretriz Brasileira de Dislipidemias e Prevenção da Aterosclerose - 2017 [Internet]. Arq Bras Cardiol. 2017;109(2 Supl 1):1-76. doi: 10.5935/ abc.20170121
23. Malachias MVB, Souza WKSB, Plavnik FL, Rodrigues CIS, Brandão AA, Neves MF, et al. $7^{\text {a }}$ Diretriz Brasileira de Hipertensão Arterial [Internet]. Arq Bras Cardiol. 2016; 107(3 Supl. 3):1-83. Disponível em: http://publicacoes.cardiol.br/2014/ diretrizes/2016/05_HIPERTENSAO_ARTERIAL.pdf

24. Oliveira JEP, Montenegro Junior RM, Vencio S, organizadores. Diretrizes da Sociedade Brasileira de Diabetes 2017-2018 [Internet]. São Paulo: Editora Clannad; 2017. Disponível em: https://www.diabetes.org.br/profissionais/images/2017/diretrizes/ diretrizes-sbd-2017-2018.pdf

25. Brasil. Ministério da Saúde. Secretaria de Atenção à Saúde. Departamento de Atenção Básica. Caderno de Atenção Básica Rastreamento [Internet]. Disponível em: http://bvsms.saude.gov. br/bvs/publicacoes/caderno_atencao_primaria_29_rastreamento. pdf

26. Lotufo PA. O escore de risco de Framingham para doenças cardiovasculares. Rev. Med. 2008;87(4):232-7. doi: 10.11606/ issn.1679-9836.v87i4p232-237

27. Viterbo LMF, Dinis MAP, Vidal DG, Costa AS. Implementation of an Interdisciplinary Approach to Promote Workers Global Health Status in the Oil Industry, Brazil (2006-2015). Int J Environ Res Public Health. 2019;16(12):2148. doi: 10.3390/ijerph16122148 